\title{
Migration of endotacker into the bladder 7 years after laparoscopic retroperitoneal burch application
}

\author{
Ahmet Salvarci ${ }^{1}$, Yunus Agrali ${ }^{2}$ \\ ${ }^{1}$ Department of Urology, Konya Hospital, Semsi Tebrizi Mh. Serafettin Caddesi, Konya, Turkey and ${ }^{2}$ \\ Department of General Surgery, Konya Hospital, Semsi Tebrizi Mh. Serafettin Caddesi, Konya, Turkey
}

\section{ABSTRACT}

Laparoscopy began to be used widely since the second half of 1990s as an alternative to laparotomy or vaginal approaches in incontinence and pelvic diseases in women, based on its claimed better success rates. Injuries were reported in the bladder, gastrointestinal system and the entry of the Verress cannula in early and late laparoscopic applications. De-novo urging, voiding dysfunctions, marked recurrences and surgical inefficiencies were observed in 5-year follow-ups after laparoscopic incontinence surgery. Although tension-free midurethral sling operations replaced open laparoscopic colposuspensions nowadays, laparoscopic colposuspension is still preferred in cases where simultaneous laparoscopic paravaginal repair or sacrocolpopexy is considered or where synthetic graft implantation is contraindicated.

Moreover, meshes and endotackers are still frequently used in many laparoscopic applications in various clinics. The migration of the tacker used in mesh fixation in a patient where retroperitoneal laparoscopic Burch was performed 7 years ago due to stress urinary incontinence and the extraction of the ossified tacker from the bladder will be presented.

\section{ARTICLE INFO}

Key words:

laparoscopy, Burch, tacker,

bladder, migration

Int Braz J Urol. 2015; 41: 382-7

Submitted for publication:

June 06, 2014

Accepted after revision:

July 17, 2014

\section{INTRODUCTION}

Although more than 100 operations have been defined as success for preventing incontinence in women, laparoscopic Burch operation was launched as the first-line option due to its high success rate and low complication rate (1). At first, it was defined as the laparoscopic Burch colposuspension procedure in 1991 (2). The fact that cystocele could also be corrected in the laparoscopic Burch operation was described as an advantage. The extraperitoneal approach was recommended more especially for patients who underwent abdominal and retro-pubic surgery and for whom a simultaneous pelvic surgery was not planned (3). It was presented as an advantageous approach due to easy entry into the Retzius spa- ce, minimum blood loss, minimum post-operative pain, shorter period of hospitalization and more rapid return to daily activities $(2,4,5)$. The method of fixing the mesh with a tacker was also defined in addition to the standard laparoscopic method and it was indicated that the success rates were lower compared to the conventional sutured method (6). Complications such as the long duration of the operation, bladder injuries, de novo urgency, voiding dysfunctions, intestine and large vessel injuries and venous thromboembolism were previously reported in groups where the laparoscopic method was applied (7). The migration of the endotacker used for fixing the mesh into the bladder in a patient in whom retroperitoneal Burch was applied 7 years ago will be presented as a different late complication. 
CASE

A 48-year old female patient with complaints of blood clots in the urine, burning during urination, frequent urination and incontinence was examined in the out-patient department. It was learned that retroperitoneal laparoscopic Burch was performed on the patient by obstetricians 7 years ago due to incontinence and bladder prolapse. The patient reported that she constantly felt urgency after the surgery. She also indicated that she had urgency type incontinence after the surgery. The patient reported that in addition to the use of gentamicin, ciprofloxacin for recurring urinary tract infections after the laparoscopic Burch procedure, and even ceftriaxone due to pyelonephritis, she received continuous anticholinergic therapy for irritative urinary complaints and ongoing incontinence. Grade II cystocele was observed at physical examination. Marshall-Marchetti-Krantz and Bonney test resulted (+). Urethral hypermobility was observed at the Q type test. The blood urea was $18 \mathrm{mg} / \mathrm{dl}$, creatinine was $1.1 \mathrm{mg} / \mathrm{dl}$ and

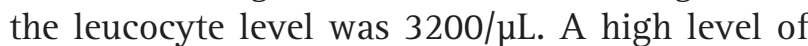
leucocytes, erythrocytes and oxalate crystals were detected in the urinalysis. E. coli $100.000 \mathrm{cfu} / \mathrm{ml}$ proliferation was observed in the urine culture. No pathology was observed in the upper urinary system at ultrasonography, while it was reported that there was a stone in the bladder (Figure-1a). Prior to the assessment of the pelvic tomography, the radiologist recorded that tackers were observed around the bladder and urethra upon being informed about laparoscopic Burch and endotackers and indicated that probably one of these was inside the bladder while another one was behind the bladder (Figures 1b, c and d). A stone adhered on the right lateral wall of the bladder was observed during cystoscopy performed on the patient with spinal anesthesia (Figure-2). When the stone was removed from the bladder wall with forceps, the metallic helical structure of its adhered part was revealed and it was understood that this was an ossified tacker utilized in laparoscopy which had migrated in the bladder (Figure-2). The tacker was extracted via forceps accompanied by cystoscopy. The integrity of the bladder wall was monitored in the control cystoscopy conducted 45 days after the cystoscopic removal of the ossified endotacker which had migrated into the bladder. Grade III cystocele was detected in the vaginal examination of the patient. The patient's mixed incontinence was verified urodynamically and vaginal anterior colpography as well as transobturator tape were performed on the patient in the same session. In the physical examination of the patient, who had no incontinence complaints after surgery, it was observed that the Marshall-Marchetti-Krantz and Bonney tests were normal and that her urethral hypermobility to the Q type test recovered. No proliferation was observed in the urine culture in the post-surgical follow-ups.

A consent was received from the patient, indicating that her data would be archived and that her post-surgery data would be used for a scientific presentation.

\section{DISCUSSION}

Laparoscopic retroperitoneal Burch colposuspension has entered into our practice for the purpose of achieving urinary continence, simultaneous repair of the cystocele and lowering morbidity. The duration of the operation which was longer in the initial cases, shortened with technical support and with experience. Bladder perforation, hypotension, pneumomediastinum and pneumothorax were reported during the procedure in the initial cases (7-9). Urgent laparotomy was per-

\section{Figure 1a - Ultrasonography image of bladder stone.}

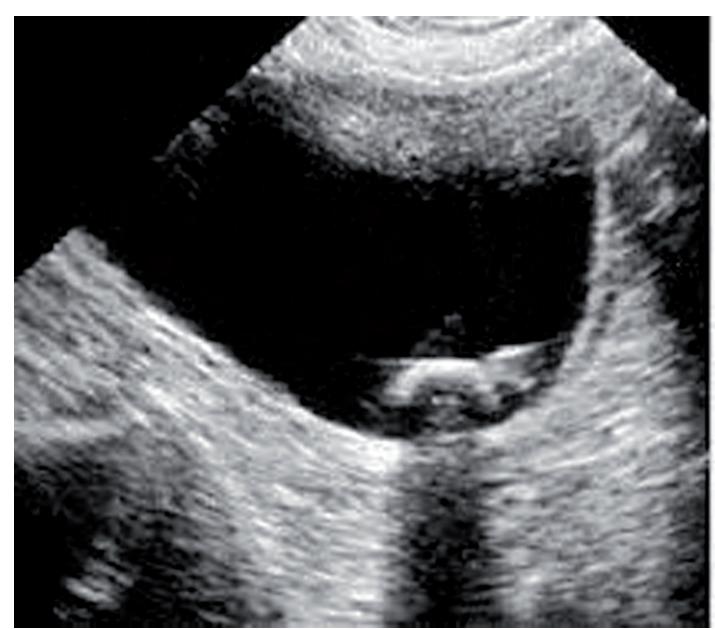


Figure 1b - Tomography (CT) image of endotacker inside the bladder.

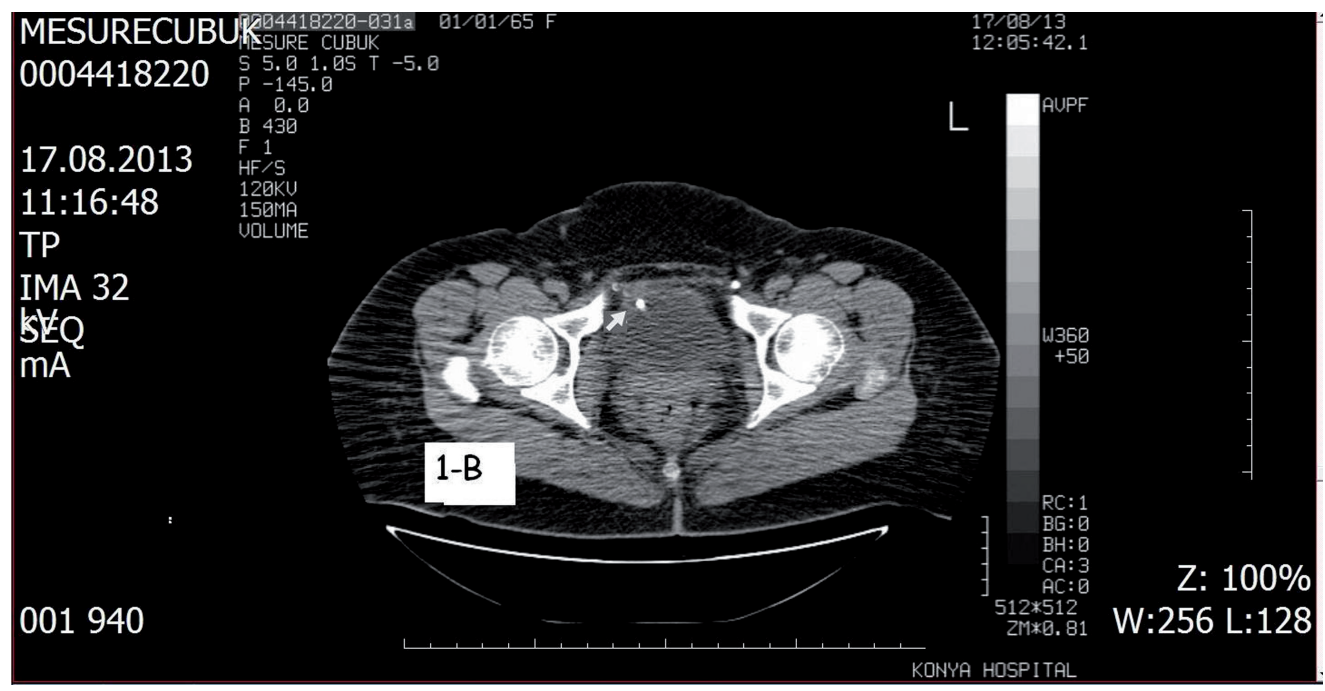

Figure 1c - CT image of normally localized endotackers.

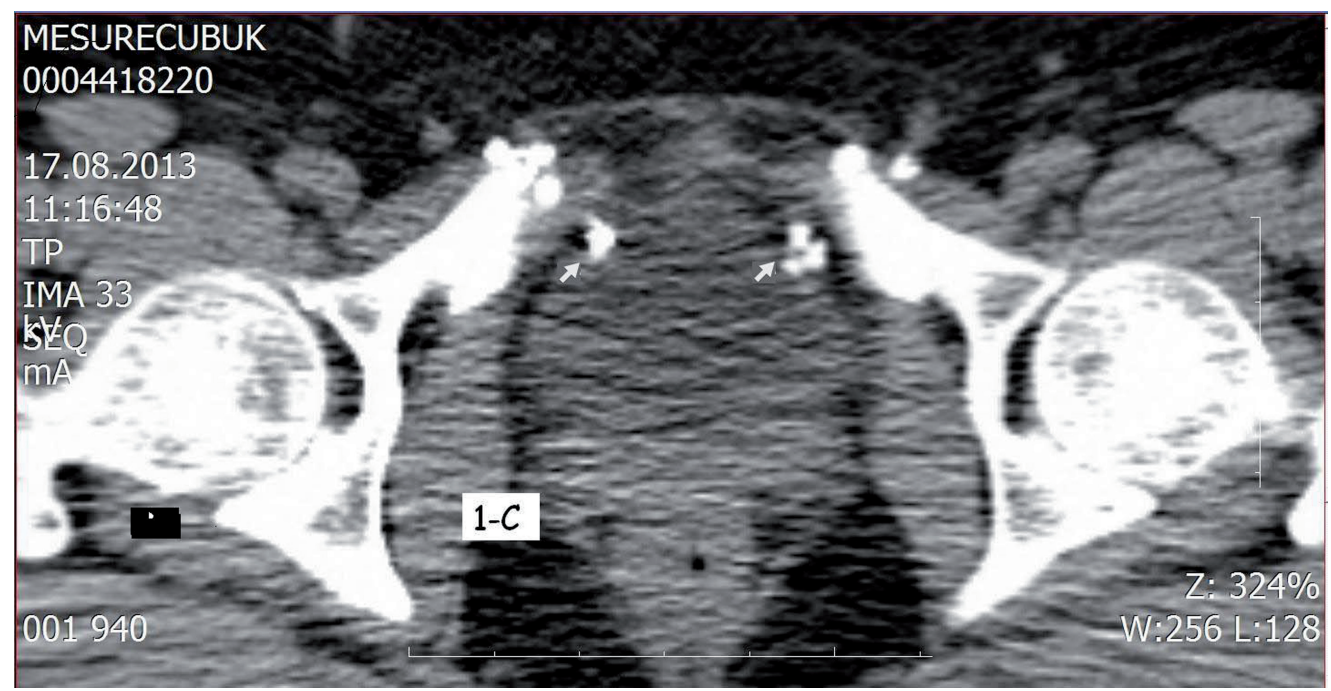

formed in complications such as bowel damage, large vessel injury and venous thromboembolism on 280 patients who underwent laparoscopic Burch (22.2\%) within the group of 1.265 patients on whom laparoscopy was performed in the multi-centric prospective case loading analysis (10). In the comparison of sutures and tackers in colposuspensions comprising 254 women, it was also claimed that tacker was more risky and that the recurrence of incontinence was higher after tacker (11). Retroperitoneal hematoma as well as blad- der injury were observed in the same patient after laparoscopic Burch performed with tacker (12). Bladder damage, hemorrhage, urinary infection, fever, sepsis, de novo urgency, subcutaneous emphysema were reported in a 800-case series where laparoscopic Burch procedure was performed (13). Tackers are used widely used in general surgery for mesh fixation at laparoscopic inguinal hernia repairs and single-incision laparoscopic surgeries are performed with the assistance of an arthroscopic cannula and tacker $(14,15)$. Iatrogenic bladder 
Figure 1d - CT image of endotacker migrated behind the bladder.

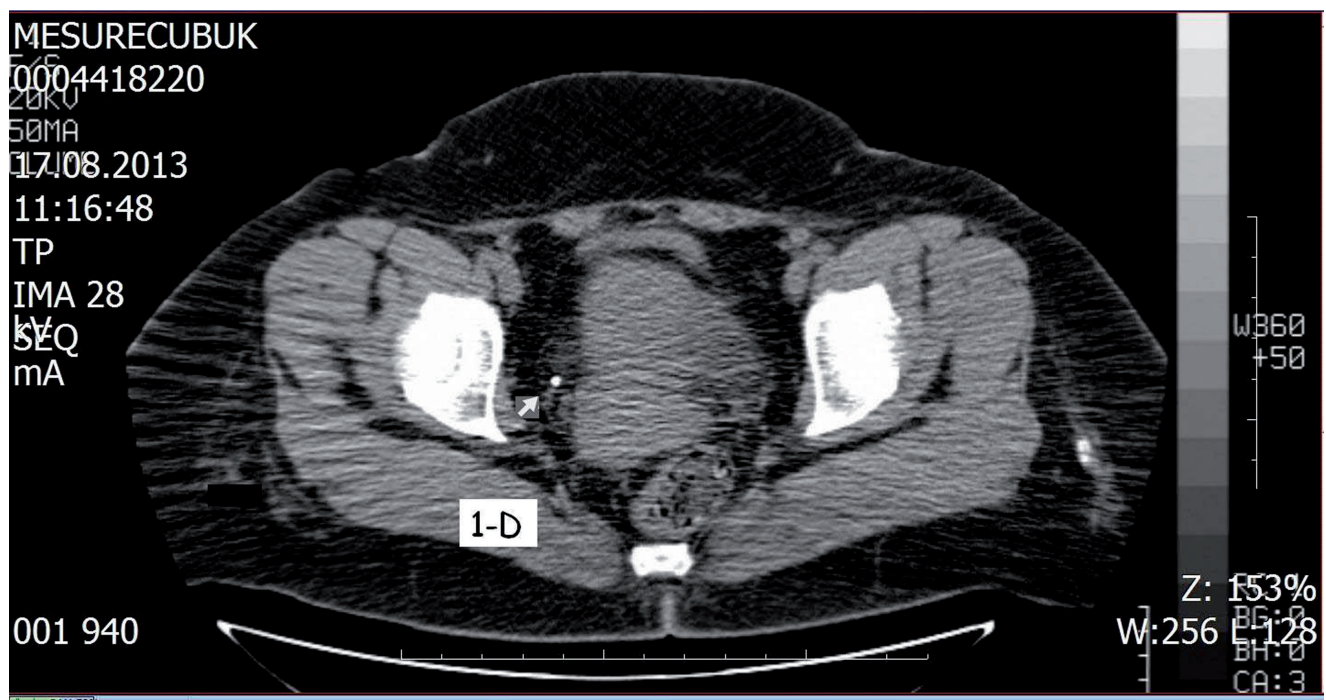

Figure 2 - Migrated tacker adhered on the bladder wall at cystoscopy and post-surgery images.

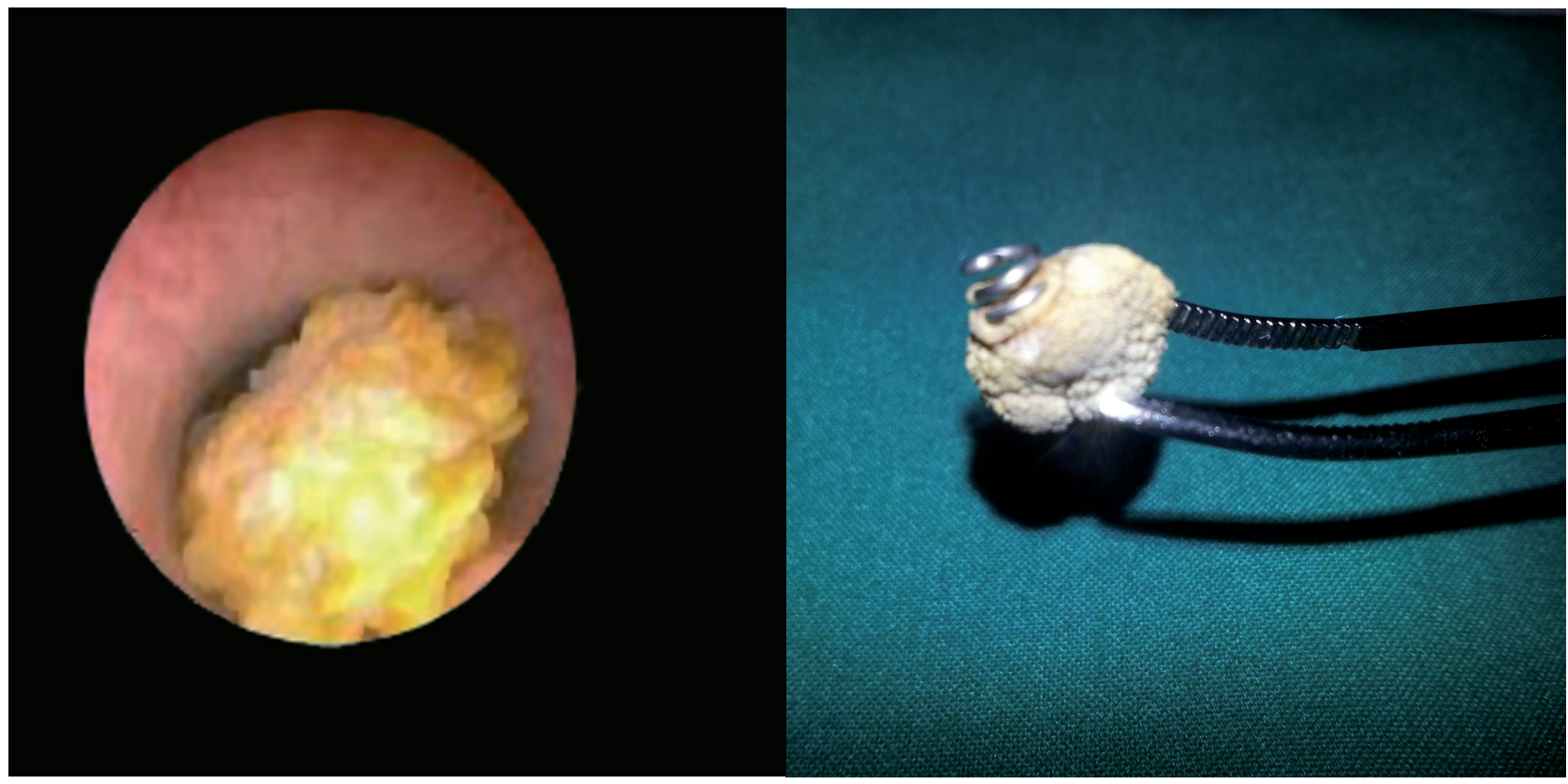

stone formation associated with non-absorbable sutures passing through the bladder after the Burch colposuspension applied again in the treatment of urinary incontinence was reported previously and dispareunia lasting 2 years as well as irritative symptoms were observed in these patients (16). It was proposed to perform cystoscopy post-sur- gically in these patients, where necessary, and to apply the sutures more laterally from the bladder.

Although the complication rates vary within the past 20 years, they were always similar in nature. The investigation of retrospective laparoscopic Burch complications did not reveal any endotacker migration into the bladder similar to 
our case. It was indicated only in one report that bladder stones on the surgical tape were removed via cystoscopy 6 years after laparoscopic colposuspension for stress incontinence occurring as a long term complication and this was stated to be the first such case (17). Although we initially thought that the tacker was placed upon directly perforating the bladder in our patient, the fact that no pathology was detected in the bladder in numerous ultrasonographies and cystoscopies performed in different centers throughout 7 years made us shy away from this thought. We were more convinced that the tacker partially perforated the bladder during the surgical procedure and entered into the bladder upon eroding with time. We believe that our thought is more substantiated with the ossified suspended tacker image on the right lateral wall of the bladder (Figure-2). Although this is a tacker whose tip is in partial contact with the bladder, the fact that it is inside the bladder supports our claim of migration. Furthermore, the fact that one of the tackers placed on the right side was outside its normal position towards the back of the bladder, gave the impression of a tacker which was either not suitably positioned or missed or again of a perivesically migrated tacker (Figure-1d). It was observed in this case that the endotacker may have migrated inside the bladder after years and it was considered that it could have been one of the late complications of laparoscopic Burch procedure.

Nowadays, tension-free midurethral slings (such as transobturator tapes) have replaced laparoscopic and open colposuspensions. Due to their easy application, minimum invasiveness, low recurrence rate in 10-year follow-ups and contribution to the correction of sphincter impairment unlike other operations, tension-free midurethral slings have become the first-line choice. It was also recorded that tension-free mid sling may be applied in the same session with anterior colporaphy in the presence of cystocele and that the success rate was further increased. However, in the consideration of laparoscopic vaginal repair or laparoscopic sacrocolpopexy or in vaginal applications where synthetic graft implantation is contraindicated in obstetrics and urology in the laparoscopic applications of various clinics, lapa- roscopic colposuspension Burch procedures and endotacker comprising kits are still used simultaneously nowadays. In the light of our clinical experience, we prefer mostly transobturator tape (TOT) and transvaginal tape (TVT) procedures in urinary incontinence surgery especially for supporting external urinary sphincter, ensuring urethral hypermobility stabilization and repairing in the same session the cystocele which determines success in such surgical procedures. It was observed in the patient that the endotacker migrated behind and inside the bladder similarly to the migration of teflon which is a synthetic material used in the treatment of vesicoureteral reflux in the past. We recommend TOT and TVT even in laparoscopic gynecological approaches if urinary incontinence is to be treated in the same session.

In conclusion, we believe that it should not be forgotten that endotackers may migrate as a late complication in radiological images at different anatomic sites including stones in the bladder of patients with a history of laparoscopic incontinence where endotackers are used.

\section{CONFLICT OF INTEREST}

None declared.

\section{REFERENCES}

1. Guner H, Yildiz A, Erdem A, Erdem M, Tiftik Z, Yildirim $M$. Surgical treatment of urinary stress incontinence by a suburethral sling procedure using a Mersilene mesh graft. Gynecol Obstet Invest. 1994;37:52-5.

2. Vancaillie TG, Schuessler W. Laparoscopic bladderneck suspension. J Laparoendosc Surg. 1991;1:169-73.

3. Frick AC, Paraiso MF. Laparoscopic management of incontinence and pelvic organ prolapse. Clin Obstet Gynecol. 2009;52:390-400.

4. Bulent Tiras M, Sendag F, Dilek U, Guner H. Laparoscopic burch colposuspension: comparison of effectiveness of extraperitoneal and transperitoneal techniques. Eur J Obstet Gynecol Reprod Biol. 2004;116:79-84.

5. Miannay E, Cosson M, Lanvin D, Querleu D, Crepin G. Comparison of open retropubic and laparoscopic colposuspension for treatment of stress urinary incontinence. Eur J Obstet Gynecol Reprod Biol. 1998;79:159-66. 
6. Zullo F, Palomba S, Piccione F, Morelli M, Arduino B, Mastrantonio P. Laparoscopic Burch colposuspension: a randomized controlled trial comparing two transperitoneal surgical techniques. Obstet Gynecol. 2001;98:783-8.

7. Radomski SB, Herschorn S. Laparoscopic Burch bladder neck suspension: early results. J Urol. 1996;155:515-8.

8. Wolf JS Jr, Carrier S, Stoller ML. Intraperitoneal versus extraperitoneal insufflation of carbon dioxide as for laparoscopy. J Endourol. 1995;9:63-6.

9. Kiilholma $P$, Mäkinen J, Chancellor MB, Pitkänen $Y$, Hirvonen T. Modified Burch colposuspension for stress urinary incontinence in females. Surg Gynecol Obstet. 1993;176:111-5.

10. Johnston K, Rosen D, Cario G, Chou D, Carlton M, Cooper M, Reid G. Major complications arising from 1265 operative laparoscopic cases: a prospective review from a single center. J Minim Invasive Gynecol. 2007;14:339-44.

11. Moehrer B, Ellis G, Carey M, Wilson PD. Laparoscopic colposuspension for urinary incontinence in women. Cochrane Database Syst Rev. 2002;(1):CD002239. Update in: Cochrane Database Syst Rev. 2006;(3):CD002239.

12. Lee CL, Yen CF, Wang CJ, Lee PS, Chiu HC. Trocar-assisted sling suspension for stress urinary incontinence: three-year follow-up. J Am Assoc Gynecol Laparosc. 2004;11:525-9.

13. Debodinance $P$, Delporte $P$, Engrand JB, Boulogne $M$. [Complications of urinary incontinence surgery: 800 procedures]. J Gynecol Obstet Biol Reprod (Paris). 2002;31:649-62.
14. Ertem M, Ozben V, Yilmaz S, Ozveri E. The use of tacker and arthroscopy cannules in SILS cholecystectomy. J Laparoendosc Adv Surg Tech A. 2010;20:551-4.

15. Sajid MS, Ladwa N, Kalra L, McFall M, Baig MK, Sains P. A meta-analysis examining the use of tacker mesh fixation versus glue mesh fixation in laparoscopic inguinal hernia repair. Am J Surg. 2013;206:103-11.

16. Moyano Calvo JL, Romero Díaz A, Ortiz Gamis A, Martínez Moran A, Castiñeiras Fernández J. latrogenic bladder lithiasis in the Burch technique. An infrequent complication. Arch Esp Urol. 2000;53:468-9.

17. Yesilli C, Seckiner I, Mungan NA, Akduman B. Stone formation on surgical staple in the bladder: a long-term complication of laparoscopic colposuspension. Surg Laparosc Endosc Percutan Tech. 2007;17:568-9.

Correspondence address: Ahmet Salvarci, MD Department of Konya Hospital Semsi Tebrizi Mh. Serafettin Caddesi, 95 Karatay, Konya, 42103, Turkey Fax: + 90505 544-2333

E-mail: yunusagrali2@hotmail.com 